\title{
Desde el compromiso social hasta el desarrollo sostenible: desafíos docentes de una educación universitaria de calidad transformadora
}

From social commitment to sustainable development: teaching challenges of a transformative quality university education

\section{Antonia Durán Ayago}

Profa. Titular de Derecho Internacional Privado.

Universidad de Salamanca, España.

Coordinadora académica de la Línea Migrantes y Derechos de la Clínica Jurídica de Acción Social

E-mail: aduran@usal.es

\footnotetext{
Resumen: Docencia, Universidad, responsabilidad social, Objetivos de Desarrollo sostenible y compromiso ético encuentran acomodo en la metodología docente del aprendizaje-servicio, método que está llamado a desempeñar un papel cada vez más importante en la formación de nuestros estudiantes universitarios. En este trabajo se aportan las principales notas características de este método y algunas claves sobre cómo orientar esfuerzos a la consecución de los ODS partiendo de la experiencia de la Clínica Jurídica de Acción Social de la Universidad de Salamanca.

Palabras clave: Responsabilidad social, Aprendizaje-servicio, Objetivos de Desarrollo Sostenible, Compromiso social, Docencia comprometida.
} 


\begin{abstract}
Teaching, University, social responsibility, Sustainable Development Goals and ethical commitment find accommodation in the teaching methodology of servicelearning, a method that is called to play an increasingly important role in the training of our university students. This work provides the main characteristic notes of this method and some keys on how to guide efforts to achieve the SDGs based on the experience of the Social Action Legal Clinic of the University of Salamanca.
\end{abstract}

Keywords: Social responsibility, Service-learning, Sustainable Development Goals, Social commitment, Committed teaching.

Sumario: 1. A modo de introducción: la responsabilidad social como guía. 2. La Agenda 2030 y el compromiso con los Objetivos de Desarrollo Sostenible. 3. Metodología de aprendizaje y servicio: objetivos y filosofía de los proyectos ApS. 4. Elementos configuradores de los proyectos de ApS. 5. Beneficios que aportan los proyectos ApS. 6. Una experiencia de aprendizaje-servicio y su articulación con los ODS: la Clínica Jurídica de Acción Social de la Universidad de Salamanca. 6.1. Contexto. 6.2. Fundamentación. 6.3. Imbricación en los ODS. 7. A modo de conclusión: la responsabilidad social como meta. 8. Bibliografía.

\title{
1. A modo de introducción: la responsabilidad social como guía
}

La responsabilidad social debe constituirse en el eje transformador de las Universidades (Ministerio de Educación, 2011). Transversalmente, debe impregnar docencia, investigación y gestión. Si una Universidad no es útil ni permeable a la sociedad en la que se inserta, entonces su misión será baldía. Simplemente, quedará desfasada y no cumplirá su función.

La Agenda 2030 y el compromiso con los Objetivos de Desarrollo Sostenible (en adelante, ODS) deben asentarse como una guía de inaplazable asunción por las Universidades (CRUE Universidades españolas). Su planteamiento no sólo implica una necesidad de acción que las Universidades están obligadas a asumir, sino una oportunidad de regeneración si son capaces de erigirse en el espacio idóneo para aunar ideas y actuación, y así servir para avanzar en un desarrollo sostenible que ha dejado de ser ya una posibilidad para convertirse en una necesidad incuestionable. 
La permeabilidad de la responsabilidad social con los ODS ha de servir de hoja de ruta también a las metodologías docentes. Su integración permitirá una comprensión crítica de la problemática social, económica y ambiental, de carácter global y local y proporcionará a nuestros estudiantes competencias en sostenibilidad, entendidas como "el conjunto complejo e integrado de conocimientos, destrezas, habilidades, actitudes y valores que capacitan para operar y transformar la realidad con criterios de sostenibilidad" (CRUE Universidades españolas). Y en este sentido adquiere una importancia cualitativa la metodología de aprendizaje-servicio sobre la que pretendo reflexionar en estas páginas, desde el prisma de la Clínica Jurídica de Acción Social de la Universidad de Salamanca, proyecto en el que participo desde su puesta en marcha durante el curso 2016/2017.

El método de aprendizaje-servicio (en adelante, ApS) es un método docente que de forma muy gráfica supone llevar la Universidad a la calle y traer la calle a la Universidad. Implica poner al estudiante en el centro de su proceso formativo, colocándolo en la posibilidad de aplicar sus conocimientos, los que ha ido adquiriendo a lo largo de su carrera, para ponerlos al servicio de una organización cuyo objetivo sea cubrir necesidades sociales. Las organizaciones del tercer sector son, por tanto, imprescindibles para este método, lo que entronca directamente con el ODS 17, puesto que permiten tejer alianzas y redes de actuación, vitales para poder desarrollar la Agenda 2030. Docentes, estudiantes y organizaciones colaboran estrechamente en un proceso de aprendizaje para el estudiante, que se transforma en servicio para la comunidad. Se trata, por tanto, de aprender enseñando, o aportando valor a su formación, el valor del compromiso social. En este proceso todos ganan y se amplifican los resultados positivos que se consiguen por parte de cada uno de los colectivos que intervienen. Para el estudiante, porque supone un proceso de verdadero empoderamiento, ya que con esta intervención en realidades concretas comprueba que los conocimientos adquiridos pueden resultar transformadores bien aplicados; para las organizaciones sociales, porque encuentran un espacio en la Universidad que les puede resultar muy útil para visibilizar sus funciones, y para los profesores porque supone una experiencia tan gratificante, que implica una fórmula para poder reconocerse de forma nítida en su vocación docente (Durán Ayago, 2019). Y, por supuesto, para la sociedad puesto que, del paso por esta experiencia, los estudiantes adquirirán habilidades y competencias que les serán útiles para el desempeño de su vida 
profesional y que contribuirán a formarlos como ciudadanos críticos y comprometidos con su entorno.

\section{La Agenda 2030 y el compromiso con los Objetivos de Desarrollo Sostenible}

En la Cumbre para el Desarrollo Sostenible, realizada en la Sede de las Naciones Unidas en Nueva York del 25 al 27 de septiembre de 2015, los Jefes de Estado y de Gobierno y Altos Representantes, reunidos en el marco del Septuagésimo Aniversario de la Organización, aprobaron la Agenda 2030 para el Desarrollo Sostenible. El documento “Transformar nuestro mundo: la Agenda 2030 para el Desarrollo Sostenible", propone un conjunto de 17 objetivos y 169 metas, cuyo fin es dar continuidad a los precedentes Objetivos de Desarrollo del Milenio, asumiendo un carácter integrado e indivisible que conjuguen las tres dimensiones del desarrollo sostenible: económica, social y ambiental.

La Agenda para el Desarrollo Sostenible es un plan de acción en favor de las personas, el planeta y la prosperidad que, a su vez, promueve la paz universal dentro de un concepto más amplio de libertad. Su magnitud se demuestra en el propósito de poner fin a la pobreza, luchar contra la desigualdad y la injusticia, hacer frente al cambio climático, haciendo realidad los derechos humanos de todas las personas. Y es el resultado de más de dos años de un intenso proceso de consultas públicas, que incluyó no sólo a representantes de gobiernos y autoridades, sino que ha sumado, en una acción sin precedentes, la opinión de toda la ciudadanía a través de un mecanismo de participación global en el cual se prestó especial atención a la opinión de los más vulnerables.

Los Objetivos de Desarrollo Sostenible están estructurados en torno a cinco vectores básicos:

- Personas: Objetivos 1 (fin de la pobreza), 2 (hambre cero), 3 (salud y bienestar), 4 (educación de calidad) y 5 (igualdad de género).

- Planeta: Objetivos 6 (agua limpia y saneamiento), 12 (producción y consumo responsable), 13 (acción por el clima), 14 (vida submarina) y 15 (vida de ecosistemas terrestres). 
- Prosperidad: Objetivos 7 (energía asequible y no contaminante), 8 (trabajo decente y crecimiento económico), 9 (industria, innovación e infraestructura), 10 (reducción de las desigualdades) y 11 (ciudades y comunidades sostenibles).

- Paz: Objetivo 16 (paz, justicia e instituciones sólidas).

- Alianza: Objetivo 17 (alianzas para lograr los objetivos).

Figura 1. Representación gráfica de los ODS

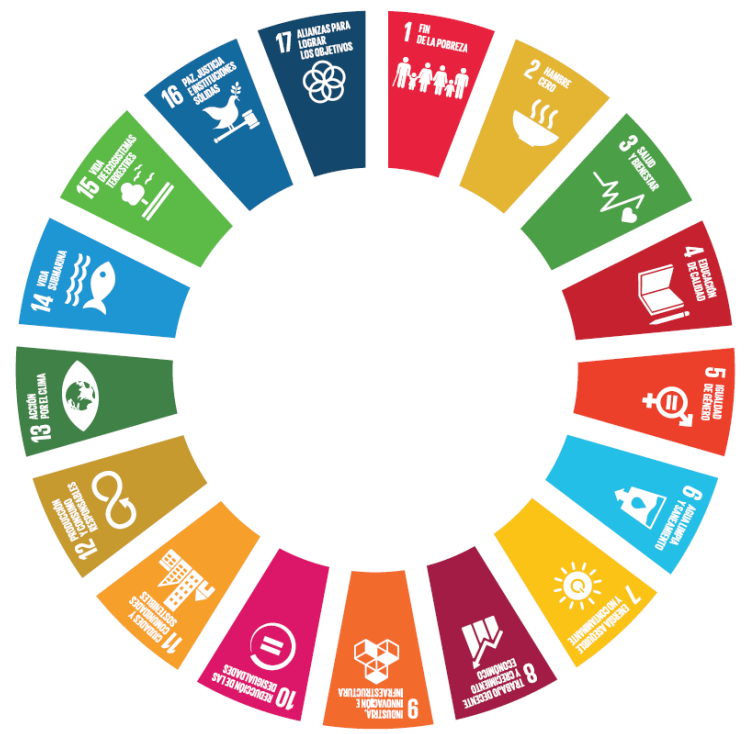

Fuente: Objetivos de desarrollo sostenible. Naciones Unidas

Desde la perspectiva universitaria, el principio de integralidad de la Agenda permite llevar a cabo una lectura transformadora de la misión, marco institucional, políticas y acciones de la Universidad que posibilite incorporar el desarrollo sostenible en el núcleo de sus decisiones y su actividad. La Universidad debe sentirse directamente interpelada por la Agenda 2030, pues ocupa una esfera privilegiada para contribuir a un desarrollo sostenible e inclusivo, no en vano la justicia, la dignidad y la igualdad de las personas debería formar parte de su ADN. Desde la perspectiva docente, la metodología de aprendizaje-servicio puede aportar el escenario idóneo para contribuir a ellos, aunando formación y compromiso con el entorno (CADEP, 2012). 


\section{Metodología de aprendizaje y servicio: objetivos y filosofía de los proyectos ApS}

El 29 de mayo de 2015 se aprobaba por el Comité Ejecutivo y el Plenario de la Comisión de Sostenibilidad (Grupo CADEP) de la Conferencia de Rectores de las Universidades españolas (CRUE), el documento técnico titulado "Institucionalización del AprendizajeServicio como estrategia docente dentro del marco de la Responsabilidad Social Universitaria para la promoción de la Sostenibilidad en la Universidad" (CADEP, 2015).

En este documento se destaca que en 2001 la Conferencia de Rectores de las Universidades españolas consideró que la Universidad debía asumir un papel protagonista en los procesos de desarrollo humano, explorando y llevando a la práctica nuevas estrategias destinadas a construir una sociedad más justa y participativa, y que la Estrategia Universidad 2015 (Ministerio de Educación, 2010), que enmarca el proceso de modernización de la Universidad española, insta a prepararse para contribuir a la promoción del nuevo modelo social y a incorporar en su modelo formativo prácticas docentes y de aprendizaje que integren adecuadamente la preparación para la práctica profesional y para el ejercicio de responsabilidad social de sus estudiantes y titulados.

El marco legal que desarrolla la responsabilidad universitaria y en la que pueden enmarcarse los proyectos de ApS se encuentra en el artículo 64.3 del Real Decreto 1791/2010 de 30 de diciembre por el cual se aprueba el Estatuto del Estudiante Universitario, según el cual, las Universidades favorecerán prácticas de responsabilidad social y ciudadana que combinen aprendizajes académicos en las diferentes titulaciones con la prestación de servicios a la comunidad, orientadas a la mejora de la calidad de vida y la inclusión social. Y en el Real Decreto 1027/2011 de 15 de julio, por el que se establece el marco Español de Cualificaciones para la Educación Superior, conocido como MECES, que tanto a nivel de Grado como a nivel de Máster prevé como un resultado de aprendizaje, la capacidad de los estudiantes de hacer reflexiones de naturaleza ética en su campo de estudio. Y la previsión de resultados de aprendizaje de esta naturaleza, supone concebir a la Universidad, también, como un espacio de aprendizaje ético, así como la necesidad de definir estrategias, como las presentes en el ApS, que lo hagan posible. 
La metodología ApS está consolidada en la educación universitaria de otras regiones del mundo, donde no sólo se desarrollan proyectos, sino que existen estructuras dentro y fuera de la Universidad que dan soporte y promueven estas acciones. En nuestro país, se trabaja el ApS en un número considerable de universidades públicas y privadas, y se están dando los primeros pasos en la institucionalización de esta metodología en algunas de ellas (Arambuzabala y otros, 2015; Chiva-Bartoll y otros, 2018; Rubio y otros, 2013; Santos Rego y otros, 2016). El ApS progresivamente está dejando de ser un conjunto de iniciativas individuales para convertirse en prácticas institucionalizadas apoyadas en estructuras más estables. Si a ello se une el factor amplificador y a su vez conductor que los ODS pueden aportar, contamos con una fórmula idónea y poderosa para contribuir al desarrollo de una formación holística y transformadora que aporte el valor del compromiso social a la educación universitaria.

La metodología ApS permite a los estudiantes aprender y desarrollarse a través de la participación en servicios comunitarios tutorizados, que se inician en el contexto de las actividades a realizar en las asignaturas de los Grados o Másteres que estén cursando, y se dirigen a satisfacer las necesidades reales del entorno, con la finalidad de mejorarlo.

Estas actividades pretenden promover el civismo, facilitar la relación entre la teoría y la práctica y dotar de sentido social y ético a los aprendizajes que realizan, poniendo especial énfasis en el análisis crítico y la comprensión de problemas y necesidades sociales, y permitiendo a los estudiantes implicarse en su resolución de forma creativa.

Integrar institucionalmente el método ApS en la docencia universitaria supone apostar por el compromiso de que ningún estudiante de Grado debería graduarse sin haber realizado una experiencia de $\mathrm{ApS}$, que implica un servicio a la comunidad, servicio que ha de estar planificado, tutelado, coordinado y ha de ser evaluado académicamente.

En este sentido, hay que destacar que los objetivos básicos de los proyectos ApS deben ser:

- Analizar y comprender problemas y necesidades sociales como estímulo de la capacidad crítica y la iniciativa solidaria.

- Desarrollar la competencia en la resolución de problemas reales de forma creativa. 
- Promover retos cívicos dirigidos a la construcción de una sociedad sostenible, más justa y solidaria.

- Posibilitar y potenciar relaciones interdisciplinares en la realización de los proyectos, que integren la complejidad real de las situaciones a las que van a tener que enfrentarse una vez comiencen su etapa profesional.

- Fortalecer el vínculo de la Universidad con otros agentes de la sociedad, desde Administraciones públicas a actores sociales pasando por empresas $\mathrm{y}$ organizaciones del tercer sector, aprovechando su experiencia en la creación y consolidación de alianzas y visibilizando el trabajo que desempeñan con colectivos vulnerables.

La forma de enseñar a nuestros estudiantes puede experimentar una singular mejora si logramos además de transferir conocimientos, sensibilizarlos acerca de la necesidad de comprometerse activamente con la sociedad. Y esta actitud de compromiso activo implica a todas las ramas de conocimiento, y, en consecuencia, a toda la docencia que impartimos como Universidad.

Esta metodología puede utilizarse en todas las titulaciones de Grado y también de Posgrado, integrarse en las programaciones docentes que aplica el profesorado en sus clases, en el marco de las prácticas externas de los estudiantes, e incorporarse en las ofertas temáticas abordadas en los Trabajos Fin de Grado y Fin de Máster. Todo el profesorado formado previamente en ApS puede tutelar estos proyectos, que han de estar relacionados con el contenido de las asignaturas que imparten y aplicarse en organizaciones sociales y en instituciones educativas (asociaciones, ONG, fundaciones, instituciones públicas, etc.).

Las materias sobre las que se puede trabajar desde esta metodología son plurales y diversas. En función del servicio que promuevan, se puede realizar la siguiente clasificación:

- Servicios a favor de un desarrollo ambiental y socialmente sostenible: acciones de protección en defensa del medio ambiente; conservación del patrimonio; prevención de riesgos; sensibilización y formación para la sostenibilidad. Desde esta perspectiva, se pueden abordar, entre otros, los ODS 3, 
$6,7,8,11,12,13,14$ y 15 . Por supuesto, el 4, educación de calidad, estaría presente en todos ellos con carácter transversal.

- Servicios de atención a personas y a colectivos cercanos con dificultades o especialmente vulnerables: intervenciones con ancianos; con menores en entornos desfavorecidos; con personas enfermas, discapacitadas o en riesgo de exclusión; con personas necesitadas de apoyo formativo, con migrantes, etc. Los ODS 1, 2, 5, 8, 10 y 16, entre otros, se podrían trabajar desde esta esfera.

- Servicios de mejora de la calidad de vida y participación ciudadana: actuaciones cívicas, de dinamización social y cultural, promoción de la educación y la salud. Se podrían trabajar desde este ángulo, entre otros, los ODS 1, 2, 3, 11 y 16.

- Servicios vinculados a problemas y necesidades surgidas en las redes sociales: privacidad, uso de las redes por menores, riesgos derivados de su uso. Los ODS $3,5,9$ y 16 , entre otros, podrían abordarse desde esta perspectiva.

- Servicios vinculados a causas solidarias y humanitarias de amplio alcance, frente a situaciones críticas, conflictos bélicos, defensa de los derechos humanos, movilizaciones frente a desastres naturales o acciones de ayuda humanitaria. Los ODS 1, 2, 5, 8, 10, 11, 13 y 16 podrían servir de base para trabajar con los estudiantes.

\section{Elementos configuradores de los proyectos de ApS}

La metodología ApS pivota sobre el análisis y la comprensión de problemas y necesidades sociales, posibilitando que los estudiantes se impliquen en su resolución, poniendo en práctica los conocimientos teóricos que han ido adquiriendo a lo largo de su formación, estimulando además su capacidad crítica y la iniciativa solidaria, promoviendo, en definitiva, el compromiso social.

En el proyecto de ApS, los estudiantes son el centro del proceso de aprendizaje, parte de ellos y se dirige a las organizaciones de carácter social, que, por otro lado, son parte esencial del proyecto, puesto que sirven para catalizar la realidad y plantear las necesidades sociales que demandan solución, proponiendo un trabajo conjunto y colaborativo con los estudiantes. 
Los elementos básicos del ApS son:

- La promoción de un aprendizaje integrado de conocimientos, habilidades, actitudes y valores, relacionados con los objetivos de aprendizaje de la materia de estudios desde la que se promueve.

- La prestación de un servicio de alta calidad para resolver necesidades sociales reconocidas por la comunidad.

- La colaboración entre estudiantes, profesores, personal de organizaciones civiles de base, empresas, ONGD y demás entidades del tercer sector. Los estudiantes desde las asignaturas (teóricas y prácticas) que están cursando, tutorizados por su profesor/a. Todo el profesorado, desde todos los Grados, también de Posgrado y asignaturas pueden tutorizar proyectos de ApS, relacionados con los contenidos explicitados en su Guía Docente. Los Proyectos han de estar relacionados con el contenido de la asignatura que cursan y aplicarse en organizaciones sociales.

- La participación activa de los estudiantes que planifican el proyecto, asumen roles y tareas apropiadas para dar respuesta a la necesidad real a solucionar; organizan las sesiones de reflexión y evaluación.

- La responsabilidad cívica de los estudiantes en la contribución que realizan a la comunidad y el impacto de sus acciones.

- La reflexión mediante el establecimiento de relaciones entre las experiencias de servicio a la comunidad y el desarrollo de los objetivos y competencias del aprendizaje académico propio de la materia de estudios desde la que se realiza el ApS.

- La evaluación que involucra a todos los participantes en el proyecto de ApS.

Es preciso destacar que el ApS no es voluntariado, dado que en este último la finalidad única es el servicio a la comunidad, mientras que en los proyectos ApS hay dos finalidades que se buscan al mismo tiempo: el servicio a la comunidad y el aprendizaje curricular que este servicio proporciona al estudiante.

El ApS no se identifica siempre con las prácticas externas curriculares. La asignatura de prácticas externas es una de las vías de encaje curricular del ApS, pero no siempre tendrán este componente. 
El ApS no se identifica por norma general con la cooperación internacional, pero puede tener este componente en función del destinatario y la naturaleza del servicio.

\section{Beneficios que aportan los proyectos ApS}

Los proyectos ApS establecen y crean sinergias positivas para todos los colectivos implicados en su puesta en marcha y desenvolvimiento. Se trata de crear simbiosis que amplifiquen las funciones de los colectivos que participen en el proyecto de ApS.

Los beneficios pueden ser múltiples y de diverso tipo. A continuación, se enumeran algunos de los potenciales beneficios en función del sector o categoría de personas implicados:

- Para el alumnado, participar en un proyecto de ApS:

1. Promueve el compromiso activo del estudiante con su comunidad.

2. Desarrolla de manera efectiva la responsabilidad social como ciudadanos y como profesionales.

3. Desarrolla de manera efectiva y evaluable competencias transversales y específicas de la titulación.

4. Incrementa la motivación por el aprendizaje.

5. Añade nuevas perspectivas en los procesos de orientación profesional.

6. Fomenta el vínculo del estudiante con su Universidad (sentido de pertenencia).

- Para el profesorado:

1. Permite la colaboración con las organizaciones sociales en el desarrollo de la docencia $\mathrm{y}$, potencialmente, añade una nueva dimensión en su investigación.

2. Permite conectar la docencia con las otras dos misiones de la universidad, especialmente con la responsabilidad social. 
3. Fomenta la creación de equipos docentes interdisciplinares.

4. Favorece el rol docente de mentor, guía o facilitador del aprendizaje que impulsa el EEES.

5. Facilita la formación y evaluación por competencias.

- Para la Universidad:

1. Permite desarrollar la tercera misión de la Universidad (compromiso con las personas, con la ciencia, con la comunidad y con los valores) y la política de responsabilidad social, favoreciendo la participación directa de toda la comunidad universitaria, potenciando, además, la transferencia del conocimiento.

2. Favorece una visión integradora de las tres misiones de la Universidad.

3. Favorece procesos de mejora y calidad docente.

4. Supone un marco privilegiado para hacer efectivo el modelo de formación integral de los estudiantes como ciudadanos, que, con su nivel cultural, sus conocimientos y habilidades específicas y su espíritu crítico han de ser la base de una sociedad más justa, solidaria, y en última instancia, sostenible.

5. Refuerza el papel de la Universidad como una institución que aporta bienestar social y económico al territorio.

- Para la sociedad:

1. Permite ofrecer soporte a colectivos y entidades sin recursos o desfavorecidos.

2. Proporciona la oportunidad para una colaboración más estrecha entre estudiantes, ciudadanos y profesionales con un fuerte compromiso social.

3. Permite estimular un desarrollo socioeconómico sostenible y materializar las metas de los ODS. 


\section{Una experiencia de aprendizaje-servicio y su articulación con los ODS: la Clínica Jurídica de Acción Social de la Universidad de Salamanca}

\subsection{Contexto}

Tal y como figura en el preámbulo del Reglamento de funcionamiento de la Clínica Jurídica de Acción Social (2017), “[1]a Clínica Jurídica de Acción Social de la Universidad de Salamanca (en adelante, la Clínica o CJAS) es un espacio de aprendizaje en el que el alumnado implicado pone en práctica los conocimientos adquiridos a lo largo de los estudios de Grado mediante la realización de unas prácticas integradas en las que actúan como si fueran "profesionales", estudiando y resolviendo asuntos prácticos reales planteados por ONGs y entidades del tercer sector bajo la coordinación y supervisión del personal docente de universidades y profesionales de diversos ámbitos. Con esta metodología se consigue, por un lado, trabajar las competencias y habilidades que debe tener todo profesional del Derecho, de la Ciencia Política y de la Criminología, enfrentándose a los dilemas éticos que pueden surgir en el ejercicio profesional y a los problemas de la realidad social, especialmente de los colectivos más desfavorecidos o en situación de vulnerabilidad, y por otro lado, se logra formar a juristas, a especialistas en Ciencia Política y en Criminología más comprometidos socialmente y sensibilizados ante las desigualdades y en la lucha contra la injusticia, es decir, con una mayor conciencia social, desempeñando así la función social que toda Universidad pública debe cumplir".

En concreto, en su artículo se indica, "[1] a Clínica Jurídica de Acción Social se integra en la Facultad de Derecho de la USAL como un espacio de servicio-aprendizaje y, a la vez, de transformación del sistema pedagógico de los estudios jurídicos, criminológicos y politológicos, así como un laboratorio de reflexión e identificación de estrategias jurídicas antidiscriminatorias mediante las cuales el alumnado puede adquirir conciencia de su importante papel en la consecución de una sociedad más justa. La lucha contra la discriminación y la exclusión social, a la vez que el fomento de la diversidad y de la tolerancia, constituyen los pilares fundamentales en los que se sustenta. La CJAS desarrollará su labor en colaboración con los grupos, asociaciones, organizaciones y redes sociales que tienen sus mismos objetivos de diseño de estrategias 
antidiscriminatorias en la búsqueda de la igualdad real y en la defensa de los derechos humanos".

La CJAS comenzó su andadura durante el curso 2016-2017, en el marco de un Proyecto de Innovación Docente concedido por el Vicerrectorado de Docencia de la Universidad de Salamanca. Durante ese curso se puso en marcha como proyecto piloto y se trabajó con 24 estudiantes procedentes de los Grados de Derecho, Ciencia Política y Administración Pública y Criminología. Durante ese periodo se conformaron los equipos en torno a cuatro iniciales líneas de actuación, incorporando a docentes de todos los Departamentos adscritos a la Facultad de Derecho de la Universidad de Salamanca. En la actualidad participan 23 profesores de 11 áreas de conocimiento diferentes y 48 estudiantes de los tres Grados citados. Las líneas de actuación son Discapacidad y dependencia; Medio ambiente y consumo responsable; Memoria histórica y democrática; Menores y uso de Internet, a las que se unieron otras dos, a partir del curso 2019/2020, Intervención penitenciaria y Derechos humanos y Migrantes y Derechos.

En la Junta de Facultad de Derecho del 13 de diciembre de 2016 se aprobó la modificación de las Normas sobre ordenación académica de los Estudios de Grado de la Facultad de Derecho de la Universidad de Salamanca, incorporando al punto IV, dedicado a las Regulaciones especiales de las asignaturas optativas y prácticas externas y Trabajo de Fin de Grado, un apartado cuarto en el que se precisa que la Clínica se integra curricularmente en los Grados de Derecho, Ciencia Política y Administración Pública y Criminología como una asignatura optativa ofertada en el octavo semestre y a través de los Trabajos Fin de Grado con 6 créditos ECTS. A partir del curso 2017/2018, la Clínica Jurídica de Acción Social ha venido impartiéndose como asignatura optativa integrada en los citados Grados.

Además, se ha introducido también como optativa en el Máster universitario de Derecho Penal. La posibilidad de llevar a cabo el Trabajo Fin de Grado o Fin de Máster conforme al método clínico, tutelado por profesores de la Clínica Jurídica, se articula a partir del curso 2018/2019. Fuimos una de las primeras Clínicas Jurídicas en España que integraron los estudios clínicos en los planes de estudios. Con carácter habitual, la Clínica Jurídica se había venido integrando en los estudios universitarios como un trabajo voluntario por parte de estudiantes y profesores, no reconocido institucionalmente. 


\subsection{Fundamentación}

Tomando como referencia el documento que elaboramos para presentar el proyecto de la Clínica Jurídica de Acción Social ante la Junta de Facultad de Derecho, el 25 de octubre de 2016, quienes promovimos la puesta en marcha de la CJAS estábamos y estamos convencidos de que la sociedad necesita profesionales que sean algo más que puros técnicos con sólidos conocimientos; de que necesitamos juristas con una conciencia social acerca de su responsabilidad en tanto operadores jurídicos, que se perciban como actores clave para la defensa y garantía del sistema de derechos y libertades de nuestra sociedad; de que necesitamos juristas con una mente abierta, con imaginación y creatividad para abordar los nuevos retos y desafíos que afrontan las sociedades contemporáneas; de que necesitamos operadores jurídicos con capacidad de empatía, solidarios y con sensibilidad hacia la situación de los más vulnerables; profesionales que pongan el Derecho al servicio de la igualdad de oportunidades y la libertad real y efectiva de todas las personas y colectivos; de que necesitamos, en definitiva, operadores jurídicos con una actitud crítica frente a los diversos poderes que marcan y determinan los aspectos esenciales de la vida de la ciudadanía.

El método de formación basado en la clínica jurídica se presenta como una herramienta idónea para formar a estos juristas, pero no sólo a ellos. También a los futuros criminólogos y politólogos que se forman en la Facultad de Derecho de la Universidad de Salamanca, en la que la Clínica se enmarca, en la medida en que muchas de las colaboraciones que llevamos a cabo precisarán el carácter interdisciplinar, siempre enriquecedor y en muchas ocasiones necesario. Este método promueve, además, la adquisición de habilidades y destrezas, capacidades y competencias generales y específicas que son esenciales para el desarrollo de la profesión jurídica, pero que conectan también con las de otras disciplinas como la Criminología o las Ciencias Políticas. Entre ellas, la argumentación, la investigación, la negociación, la toma responsable de decisiones, la realización de entrevistas y el establecimiento de relaciones con el cliente, la elección entre estrategias, la comunicación, la expresión oral, la redacción de escritos o informes, etc. Potencia, además, la actitud reflexiva y crítica de los estudiantes en relación con la legislación vigente y la actuación de los operadores jurídicos. En tanto los estudiantes son los principales responsables de los resultados del trabajo clínico, se fomenta la autorreflexión sobre su propia actuación y sus 
consecuencias, reflexión que tiene una dimensión eminentemente ética y propicia la adquisición de una deontología profesional. Esta reflexión produce un proceso de aprendizaje más profundo en la medida en que se plantea al hilo de una situación real y no en abstracto.

El método clínico se aproxima al Derecho (y por extensión, a las otras ciencias) desde una perspectiva novedosa en relación con la concepción predominante del fenómeno jurídico. En esta aproximación es posible destacar las siguientes dimensiones:

- El Derecho como argumentación. La educación clínica se aleja de la concepción dogmática y mecanicista del fenómeno jurídico y de la labor de los juristas. Fomenta la comprensión y el uso argumentativo del Derecho como una herramienta orientada a tomar y a justificar decisiones jurídicas de las que son responsables los operadores que las adoptan.

- El Derecho conectado con la realidad y el contexto. El aprendizaje clínico promueve el acercamiento del Derecho a la realidad. El Derecho no se concibe como un conjunto de principios y normas abstractas desvinculados del entramado social y cultural, sino que se percibe como un fenómeno condicionado por el contexto y, por tanto, susceptible de ser moldeado, especificado y reformado de acuerdo con la particular realidad en la que ha de ser aplicado.

- El Derecho como elemento transformador e integrador. El método clínico rechaza también la visión conformista del Derecho como un medio para preservar el status $q u o$, estableciendo reglas, principios e instituciones que se perciben como inherentemente justos. Frente a esta posición complaciente, la perspectiva clínica concibe al Derecho como un elemento capaz de promover el cambio social, reflexionándose acerca de cómo debería ser reformado e implantado para lograr este objetivo. Frente al Derecho excluyente que obstaculiza el uso de los mecanismos jurídicos por parte de toda la población, el método clínico convierte al Derecho en un elemento integrador que potencia el acceso a la justicia en igualdad de condiciones.

- El Derecho al servicio de la sociedad y del interés público. La educación clínica contribuye a derrumbar la concepción del Derecho como una herramienta hostil y ajena a los problemas reales de la ciudadanía. Esta metodología promueve una imagen del Derecho como un mecanismo adecuado para proteger los derechos y 
el interés general y una percepción de sus operadores como profesionales accesibles y cercanos a sus conciudadanos.

Conscientes de la importancia que tiene formar juristas, politólogos y criminólogos comprometidos socialmente, la Facultad de Derecho de la Universidad de Salamanca se incorporó a las Facultades que en España ya habían puesto en práctica con éxito este método de enseñanza. Considerábamos entonces que podía constituir un impulso importante para nuestra Facultad y por extensión también para la Universidad de Salamanca.

Nuestro objetivo era traer la realidad al conocimiento y llevar el conocimiento a la sociedad, en un equilibrio bidireccional en que ganamos todos, estudiantes, profesores, profesionales y sociedad. Como miembros de una Universidad pública, éramos y somos conscientes de que nuestro deber tiene que encontrar acomodo en el compromiso social. Sólo así puede alcanzar valor efectivo y pleno la función que corresponde a la Universidad.

En estos cinco años de andadura, los objetivos que hemos perseguido, más en concreto, han sido:

1. Promover y mejorar el aprendizaje activo y la formación práctica del alumnado de la Facultad de Derecho mediante el desarrollo de habilidades profesionales, profundizando en el aprendizaje autónomo promovido por Bolonia.

2. Contribuir a la formación de profesionales críticos con el sistema y sensibles a la idea de justicia social en el actual contexto sociopolítico.

3. Potenciar un contacto directo con la realidad social objeto de la intervención profesional.

4. Incentivar el esfuerzo como presupuesto del éxito.

5. Profundizar en el aprendizaje colaborativo y en una formación holística del alumnado.

6. Promover en el alumnado la conciencia social, los valores éticos y el compromiso en la defensa de los derechos humanos.

7. Potenciar y mejorar la transferencia a la sociedad del conocimiento y de la investigación generados en la Universidad. 


\subsection{Imbricación en los $O D S$}

Todas las Clínicas Jurídicas trabajamos de una u otra manera en el desarrollo de los ODS, aunque es verdad que no todas lo hemos explicitado con claridad hasta el momento en nuestras memorias. En la CJAS las temáticas que trabajamos son variadas, pero tienen como común denominador prestar atención a distintos colectivos que se caracterizan por su especial vulnerabilidad. En este momento, las líneas de actuación que desarrollamos son: Discapacidad y dependencia; Intervención penitenciaria y Derechos humanos; Medio ambiente y consumo responsable; Memoria histórica y democrática; Menores y uso de Internet y Migrantes y Derechos. Todas ellas las trabajamos con estudiantes del Grado en Derecho (Guía académica, curso 2020/2021), Ciencia Política y Administración Pública (Guía académica, curso 2020/2021), y Criminología (Guía académica, curso 2020/2021). En la Clínica jurídico-penal, integrada como asignatura optativa en el Máster de Derecho Penal de la Universidad de Salamanca (Guía académica, curso 2020/2021), se van trabajando distintos temas en cada curso, en función de las peticiones de colaboración que nos llegan. En el seno de la Clínica jurídico-penal, se elaboró, por ejemplo, el informe por los alumnos de la promoción 2017/2018, que ha conseguido que se haga justicia en el asesinato de los jesuitas en El Salvador (Radio Usal, 2018).

Figura 2. Compromiso de la Clínica jurídica de Acción social
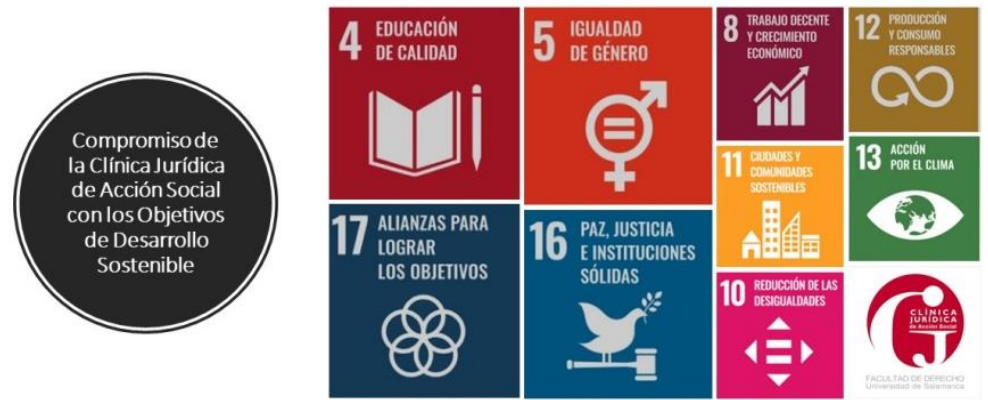

Fuente: Objetivos de desarrollo sostenible. Naciones Unidas

A continuación, intentando realizar un ejercicio exegético, explicaré cómo considero que desde la CJAS contribuimos a desarrollar algunos de los ODS 
Figura 3. Objetivo 4

EDUCACIÓN DE CALIDAD

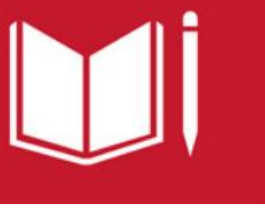

El Objetivo 4. Garantizar una educación inclusiva y equitativa de calidad y promover oportunidades de aprendizaje permanente para todos es el objetivo por antonomasia en el ámbito de la educación. En concreto, nos interesa en la CJAS dotar a nuestros estudiantes de las competencias y habilidades necesarias para que se desempeñen como profesionales empáticos y solidarios, que sean capaces de encontrar siempre la respuesta jurídica, política, criminológica, que favorezca al más vulnerable. Y si hubiera que elegir dentro de este objetivo la medida concreta en que desarrollamos mayor número de actividades y competencias sería ésta:

“4.7 De aquí a 2030, asegurar que todos los alumnos adquieran los conocimientos teóricos y prácticos necesarios para promover el desarrollo sostenible, entre otras cosas mediante la educación para el desarrollo sostenible y los estilos de vida sostenibles, los derechos humanos, la igualdad de género, la promoción de una cultura de paz y no violencia, la ciudadanía mundial y la valoración de la diversidad cultural y la contribución de la cultura al desarrollo sostenible."

Figura 4. Objetivo 5

IGUALDAD

DE GÉNERO

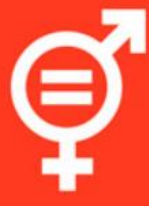

El Objetivo 5. Lograr la igualdad de género y empoderar a todas las mujeres y las niñas es transversal a todas las líneas. Partimos de la premisa de que sin igualdad no existen valores esencialmente democráticos. Específicamente, en nuestra trayectoria hemos trabajado sobre mujeres y discapacidad y también el rol de género en el uso de las tecnologías, en especial, los comportamientos opresivos que sobre las menores de edad se pueden ejercer a través de las redes sociales o del uso de los smartphones.

Figura 5. Objetivo 8

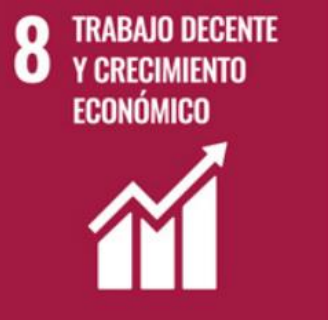

El Objetivo 8. Promover el crecimiento económico sostenido, inclusivo y sostenible, el empleo pleno y productivo y el trabajo decente para todos lo hemos trabajado fundamentalmente en la línea de Medio ambiente y consumo responsable, con carácter particular desde la importancia de desarrollar una responsabilidad social corporativa que se comprometa con el respeto íntegro de los derechos de los trabajadores y en la línea de Migrantes y Derechos, potenciando los discursos que miran 
a la migración como una oportunidad para la España vaciada y analizando las fórmulas jurídicas adecuadas para que los inmigrantes puedan disfrutar del derecho al trabajo en condiciones dignas. También en la línea de Intervención penitenciaria y Derechos humanos, desde la perspectiva de la importancia del trabajo para una eficaz reinserción y resocialización de las personas que han estado privadas de libertad.

Figura 6. Objetivo 10

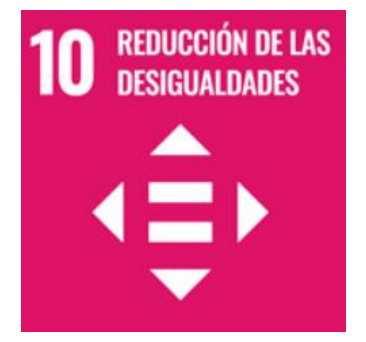

\section{El Objetivo 10. Reducir la desigualdad en los países y entre ellos}

lo hemos desarrollado especialmente en la línea de Discapacidad y dependencia, de Intervención penitenciaria y Derechos humanos y también en la de Migrantes y Derechos. En relación con la medida 10.3 Garantizar la igualdad de oportunidades y reducir la desigualdad de resultados, incluso eliminando las leyes, políticas y prácticas discriminatorias y promoviendo legislaciones, políticas y medidas adecuadas a ese respecto, hemos realizado informes, especialmente en Migrantes y Derechos, denunciando prácticas burorrepresivas y proponiendo, bien la incorporación en España de Directivas europeas que garantizan los derechos de los solicitantes o peticionarios de protección internacional en nuestro país, bien solicitando llevar a cabo una interpretación igualitaria que permita a los extranjeros en situación irregular acceder a las prestaciones de urgencia social.

Figura 7. Objetivo 11

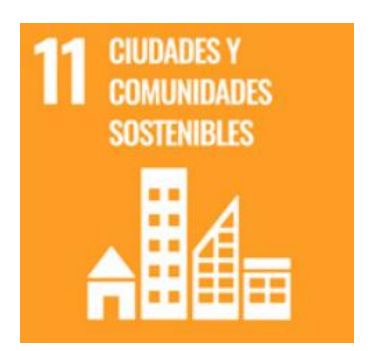

El Objetivo 11. Lograr que las ciudades y los asentamientos humanos sean inclusivos, seguros, resilientes y sostenibles lo hemos abordado en varias líneas de actuación: Discapacidad y dependencia, Medio ambiente y consumo responsable; Intervención penitenciaria y Derechos humanos y Migrantes y Derechos. También en cierto modo, en la línea de Menores y uso de Internet. Desde los colectivos vulnerables con los que trabajamos hemos abogado por el acceso a los servicios básicos adecuados, seguros y asequibles; hemos puesto en marcha campañas de reciclaje, como la recogida de tapones, con el objetivo de concienciar sobre el cambio climático; hemos trabajado fórmulas de integración de los inmigrantes en nuestras ciudades o hemos analizado la mejor manera de integrar a personas que han estado privadas de libertad en la sociedad. 
Figura 8. Objetivo 12

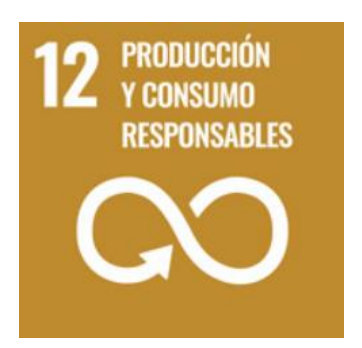

El Objetivo 12. Garantizar modalidades de consumo y producción sostenibles es el objetivo básico de la línea de Medio ambiente y consumo responsable. Desde ella se ha promovido la economía circular, se ha concienciado acerca de la necesidad de reducir el desperdicio de alimentos; se han puesto en marcha campañas de reciclaje o se han realizado seminarios formativos sobre la responsabilidad social de las empresas.

Figura 9. Objetivo 13

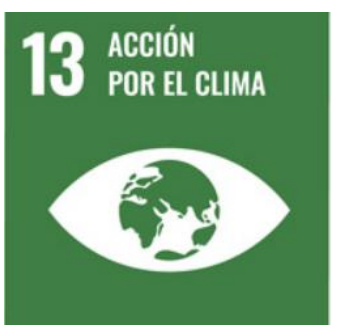

\section{El Objetivo 13. Adoptar medidas urgentes para combatir el} cambio climático y sus efectos también entra de lleno en las actuaciones llevadas a cabo por la línea de Medio ambiente y consumo responsable. Destacando el valor de sus campañas de sensibilización y divulgación sobre el cambio climático, con colaboraciones con distintas asociaciones entre ellas, Fridays for Future.

Figura 10. Objetivo 16

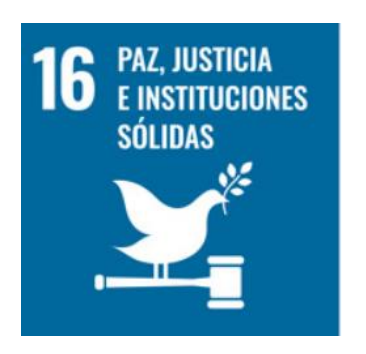

El Objetivo 16. Promover sociedades pacíficas e inclusivas para el desarrollo sostenible, facilitar el acceso a la justicia para todos

Figura 11. Objetivo 17

ALIANZAS PARA LOGRAR LOS OBJETIVOS

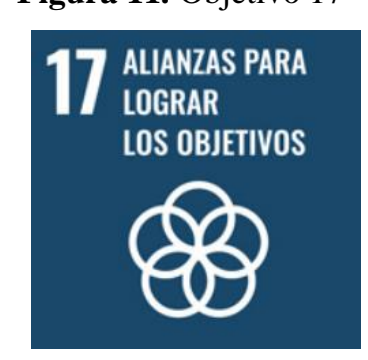

y construir a todos los niveles instituciones eficaces e inclusivas que rindan cuentas es transversal a todas las líneas por el carácter fundamentalmente jurídico que inspira a la CJAS.

El Objetivo 17. Fortalecer los medios de implementación y revitalizar la Alianza Mundial para el Desarrollo Sostenible lo hemos tenido presente desde nuestro origen, llevando a cabo convenios con distintas organizaciones del tercer sector, con colegios, institutos u otros entes con los que colaboramos. Para la CJAS es muy importante el contacto directo con la realidad que tienen que analizar nuestros estudiantes, y este contacto lo conseguimos a través de estas organizaciones que a pie de calle conocen perfectamente los problemas de los colectivos en los que intervenimos. Como se puede apreciar en nuestras Memorias de actividades, son más de 30 entidades y organizaciones las que nos acompañan y facilitan nuestra labor formativa (Blog Clínica Jurídica de Acción Social). 


\section{A modo de conclusión: la responsabilidad social como meta}

La Universidad ocupa un lugar estratégico para contribuir al cumplimiento de los ODS. Imbricados en la misión de la responsabilidad social, la Agenta 2030 debería impregnar toda la acción de la Universidad. Desde la docencia, la metodología del aprendizajeservicio aporta un medio idóneo para unir formación, compromiso y acción.

Son cada vez más numerosas las experiencias de ApS que se ponen en marcha en las Universidades españolas, pero todavía son pocas las que han optado por su institucionalización, factor clave para impulsar esta metodología integrada como una práctica de innovación docente en todos los títulos universitarios. Partiendo de la base de que los proyectos de ApS pueden llevarse a cabo en el contexto de todas las titulaciones, el compromiso en el desarrollo de la Agenda 2030 puede suponer el escenario idóneo para avanzar en la implementación de este tipo de docencia en la Universidad. Los estudiantes que participan en proyectos de ApS aportan a su formación el compromiso social y ético con los problemas del entorno, que contribuyen a solucionar o a proponer medidas que reviertan positivamente en los colectivos implicados.

Muchas de las iniciativas de ApS en las que se venía trabajando ya en las Universidades tienen ahora el reto de visibilizar de forma más específica el compromiso con los ODS en sus memorias. Si somos capaces de seguir aportando valor a la formación de nuestros estudiantes y el efecto contagio entre titulaciones logra sensibilizar a las Universidades acerca de la necesidad de su institucionalización, habremos conseguido desde la docencia maximizar la misión de la responsabilidad social, aportando prácticas docentes innovativas y de calidad que redundarán en el bien de la sociedad en su conjunto. Este desafío, al alcance de la mano, debiera plantearse como objetivo irrenunciable de las Universidades en un futuro inmediato.

\section{Bibliografía}

ARAMBURUZABALA, P., OPAZO, H., GARCÍA-GUTIÉRREZ, J. (eds.) (2015), El Aprendizaje-servicio en las universidades, UNED. 
ASAMBLEA GENERAL DE NACIONES UNIDAS (2015), Transformar nuestro mundo: la Agenda 2030 para el Desarrollo Sostenible, Accesible en https://www.agenda2030.gob.es/recursos/docs/APROBACION_AGENDA_2030.pdf

CHIVA-BARTOLL, O., GIL-GÓMEZ, J. (eds.) (2018), Aprendizaje-servicio universitario Modelos de intervención e investigación en la formación inicial docente, Octaedro.

CADEP (2012). Orientaciones para la introducción de la sostenibilidad en el curriculum. Anexo a, Directrices para la Introducción de la Sostenibilidad en el Curriculum CRUE, 2005, Declaración institucional. Grupo de trabajo Sostenibilización Curricular, Valencia: CADEP-CRUE. Accesible en http://angelsull.es/sostenibilidad/wpcontent/uploads/2013/04/Directrices-Sostenibilidad-curriculum-CRUE.pdf

CADEP (2015), Institucionalización del Aprendizaje-Servicio como estrategia docente dentro del marco de la Responsabilidad Social Universitaria para la promoción de la Sostenibilidad en la Universidad, Accesible en https://www.uv.es/uvsostenible/CRUE/2.\%20APROBADA\%20INSTITUCIONALIZA CION\%20ApS.pdf.

CLÍNICA JURÍDICA DE ACCIÓN SOCIAL (2016), Documento de presentación del proyecto de la Clínica Jurídica de Acción Social ante la Junta de Facultad de Derecho, el 25 de octubre de 2016. Accesible en https://diarium.usal.es/clinicajuridica/files/2016/11/CL\%C3\%8DNICAJUR\%C3\%8DDICA-DE-ACCI\%C3\%93N-SOCIAL-USAL-Junta-de-Facultad-25-X2016.pdf

CLÍNICA JURÍDICA DE ACCIÓN SOCIAL (2017), Reglamento de funcionamiento de la Clínica Jurídica de Acción Social, Accesible en https://diarium.usal.es/clinicajuridica/files/2016/11/Reglamento-Funcionamiento-CJAS2017.pdf.

CRUE Universidades españolas, "El compromiso de las Universidades españolas con la Agenda 2030", accesible en 
http://www.exteriores.gob.es/Portal/es/SalaDePrensa/Multimedia/Publicaciones/Docum ents/CRUE\%20Universidades\%20Espa\%C3\%B1olas.\%20Posicionamiento\%20Agenda \%202030.pdf, consultado el 23 de marzo de 2021.

DURÁN AYAGO, A. (2019), Docencia de calidad con compromiso social y el aprendizaje-servicio como medio, en https://diarium.usal.es/aduran/2019/07/12/docencia-de-calidad-con-compromiso-socialy-el-aprendizaje-servicio-como-medio/.

MINISTERIO DE EDUCACIÓN (2010), Estrategia Universidad 2015. Accesible en https://sede.educacion.gob.es/publiventa/PdfServlet?pdf=VP14872.pdf\&area=E.

MINISTERIO DE EDUCACIÓN (2011), La responsabilidad social de la Universidad y el desarrollo sostenible. Ministerio de Educación España, Subdirección General de Documentación. Accesible en https://sede.educacion.gob.es/publiventa/laresponsabilidad-social-de-la-universidad-y-el-desarrollo-sostenible/universidadespana/14925.

RED ESPAÑOLA EN CLÍNICAS JURÍDICAS, en http://clinicasjuridicas.blogspot.com/p/presentacion.html\#: :text=La\%20Red\%20Espa\%C3\%B1ola\% 20de\%20Cl\%C3\%ADnicas,de\%20la\%20que\%20se\%20benefician

RUBIO, L., PRATS, E., GÓMEZ, L. (coord.) (2013), Universidad y sociedad. Experiencias de aprendizaje servicio en la universidad, ICE. Universitat de Barcelona.

SANTOS REGO, M. A., SOTELINO LOSADA, A., LORENZO MOLEDO, M. (eds.) (2016), Aprendizaje-Servicio e innovación en la universidad. Actas del VII Congreso Nacional y II Internacional de aprendizaje-servicio universitario, Santiago de Compostela, 13-15 de octubre de 2016, Servicio de Publicaciones, Universidad Santiago de Compostela. 